Review

\title{
Kampo Medicine for Older People: A Review
}

Tatsuya Nogami ${ }^{1}$, Makoto Arai ${ }^{1}$, Koh Iwasaki ${ }^{2, *}$

1. Department of Kampo Medicine, Tokai University, School of Medicine, Isehara, Kanagawa, Japan; E-Mails: t-nogami@tsc.u-tokai.ac.jp; arai@tokai-u.jp

2. Natori-Kumanodoh Hospital, Natori, Miyagi, Japan; E-Mail: iwasaki.koh67@gmail.com

* Correspondence: Koh Iwasaki; E-Mail: iwasaki.koh67@gmail.com

Academic Editor: Christopher Maylahn

Special Issue: Age-Related Chronic Diseases

OBM Geriatrics

2022, volume 6, issue 1

doi:10.21926/obm.geriatr.2201191
Received: January 12, 2022

Accepted: February 20, 2022

Published: February 28, 2022

\begin{abstract}
Several countries have entered the super-aging era. In Japan, the recently reported average life expectancy is approximately 90 years. Kampo medicine (a form of traditional Japanese medicine) is a part of the official medical service in Japan and is widely used for treating various illnesses of older people. However, although general physicians often prescribe Kampo preparations, most Japanese general physicians do not have an intimate understanding of the iatrology of Kampo. Kampo is based on traditional Chinese medicine modified according to the Japanese environment, characteristics, and society. Although Kampo prescriptions are beneficial in geriatric medicine, they were initially developed and applied according to the medical principles of traditional Japanese and Chinese medicine. Moreover, improper administration of these traditional preparations can lead to severe adverse consequences. In this review, we have described the efficacy of Kampo medicine for geriatric syndromes and the traditional meanings and rules underlying Kampo prescriptions.
\end{abstract}

\section{Keywords}

Kampo; traditional Chinese medicine; geriatric syndrome; older people; dementia; aspiration pneumonia; behavioral and psychiatric symptom of dementia; constipation

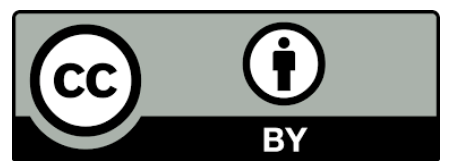

(C) 2022 by the author. This is an open access article distributed under the conditions of the Creative Commons by Attribution License, which permits unrestricted use, distribution, and reproduction in any medium or format, provided the original work is correctly cited. 


\section{Introduction}

Currently, advanced countries, including Japan, have a rapidly aging population. The average life expectancy in Japan is approximately 90 years. Kampo medicine (a form of traditional Japanese medicine) is an official medical service provided in Japan that is widely applied for treating ailments affecting older people. However, general physicians often use Kampo prescriptions without considering the iatrology of Kampo procedures. Kampo is based on traditional Chinese medicine (TCM), which was modified according to the Japanese environment, characteristics, and society. Kampo prescriptions are crucial in geriatrics and were developed following the medical principles underlying traditional Japanese medicine and TCM. Incorrect application of Kampo prescriptions can lead to severe adverse outcomes. Therefore, in this review, we described the efficacy of Kampo medicine for geriatric syndromes and the traditional meanings and protocols underlying Kampo prescriptions.

\subsection{Our Standpoint}

Kampo medicine was derived from TCM and modified according to Japanese conditions. There are numerous schools (styles or streams) of Kampo medicine. The Chinese government integrated these numerous streams into a systematic method, which is termed TCM or “中医学 (ZhongYiXue)" in Chinese. Therefore, TCM reports written in English are often based on the integrated TCM system. However, attempts to unify various streams of Kampo medicine in Japan have been unsuccessful. Hence, it is crucial to understand the style being described by the author while reading medical literature on Kampo.

Briefly, there are at least three styles of Kampo medicine, which include the traditional style (represented as 古方派 [kohōha] in Japanese), the relatively newer style (後世派 [Goseiha] in Japanese), and the modern style (there is no specific Japanese name for this style). This review focused on the modern style of integrating traditional and modern medicine, which resembles modern TCM.

\subsection{Orthography of Traditional Technical Terms, Authors' Names, and Prescriptions}

As this review describes traditional medicines, several traditional technical terms, some historically famous people and their textbooks, traditional prescriptions, and herbs have been mentioned; spelling or pronouncing some of these words in English might be difficult. For example, we refer to the important classical textbook '黄帝内経', which is pronounced as Kouteidaikei in Japanese, Huangdi Neijing in Chinese, Huangdi Neijing in Vietnamese, and Hwangjenaegyeong in Korean (we could not pronounce this spelling; an expert on Korean traditional medicine, who taught us the pronunciation, enunciated it as Huandinejen). Although using English translations in this review is necessary, using only the word "Kouteidaikei" to describe this book could be problematic since only Japanese readers would understand it. Moreover, if we wished to write a review solely for the Japanese, the Japanese language would have been better suited for writing this review. Furthermore, we are not interested in the turf war among countries regarding traditional medicines. Therefore, in this review, we used Japanese pronunciation and Chinese characters, such as 
Kouteidaikei (黄帝内経), since Chinese is the lingua franca among traditional East Asian medical experts. All Chinese, Japanese, Korean, Vietnamese, Taiwanese, and other experts can read and understand Chinese characters 黄帝内経; however, there are differences in pronunciation. There are slight differences in the characters used in each country. For example, Kouteidaikei is written as 黄帝内経 in Japan, 黄帝內経 in Taiwan, and 黄帝内经 in mainland China and Singapore. However, these slight differences are not problematic. Experts in their respective countries can read 黄帝内 経. The names of individuals are written in their native language. Finally, the names of herbs are mentioned according to their Latin nomenclature found in the Japanese Pharmacopoeia, $17^{\text {th }}$ edition [1].

\section{Geriatric Syndromes}

\subsection{Geriatric Syndromes in Modern Medicine}

Aging causes frailty and the development of geriatric syndromes, including dementia, depression, delirium, incontinence, vertigo, falls, spontaneous bone fractures, failure to thrive, neglect, and abuse [2].

Geriatric syndromes are associated with reduced life expectancy and can interfere with daily life. This review describes the efficacy of Kampo medicine for geriatric syndromes, especially dementia, behavioral and psychological symptoms of dementia (BPSD), aspiration pneumonia, and constipation. The guidelines of the Japan Geriatric Society published in 2015 have recommended the use of Kampo prescriptions for these conditions [3].

\subsection{Geriatric Syndromes or Aging Based on Kampo Medicine}

The famous medical textbook, Kouteidaikei (黄帝内経) [4], which was written between the first and second century $\mathrm{BC}$, describes the following aging-related changes in people: 1) thinning of the face; 2) hair and tooth loss; 3) hearing impairment; 4) deterioration of vision; 5) immune deficiency; 6) amnesia, short-temper, insomnia, day and night reservation, and dementia; 7) altered taste, loss of appetite, and constipation; 8) pain and numbness in the hips and knees, scoliosis, tremor causing gait disturbances, and difficulty in holding posture; and 9) sexual disturbances.

As shown above, Kouteidaikei (黄帝内経) extensively described aging, indicating that Chinese people practiced gerontology over 2,000 years ago. We have discussed several Kampo prescriptions for the symptoms of geriatric syndromes.

\section{Kampo Prescriptions for Geriatric Syndromes}

\subsection{Hachimijiogan (八味地黄丸) for Dementia}

Hachimijiogan is a Kampo prescription that was described in the Chinese textbook, Kinkiyoryaku (金匱要略), written by Zhang Zhongjing (張仲景) in the second century [5, 6]. It is used for treating middle-aged and older individuals, especially the latter, who experience loss of strength, fatigue, feeling cold, lumbago, oliguria, or frequent urination. Information on Hachimijiogan and the other medicines discussed in this review are available on the STORK website (http://mpdb.nibiohn.go.jp/stork/). Hachimijiogan comprises eight herbs, including Rehmanniae Radix, Corni Fructus, Dioscoreae Rhizoma, Alismatis Tuber, Poria, Moutan Cortex, Cinnamomi Cortex, 
and Aconiti Radix Processa [7]. The quality of these herbs is regulated by the Japanese Pharmacopoeia [1].

\subsubsection{Effects of Hachimijiogan on Cerebral Blood Flow in Patients with Dementia}

The single-photon emission computed tomography images of cerebral blood flow in a patient with severe Alzheimer's disease (AD) at baseline and after two months of hachimijiogan treatment are shown in Figure 1. The figure was taken from the study conducted by Iwasaki et al. [8].

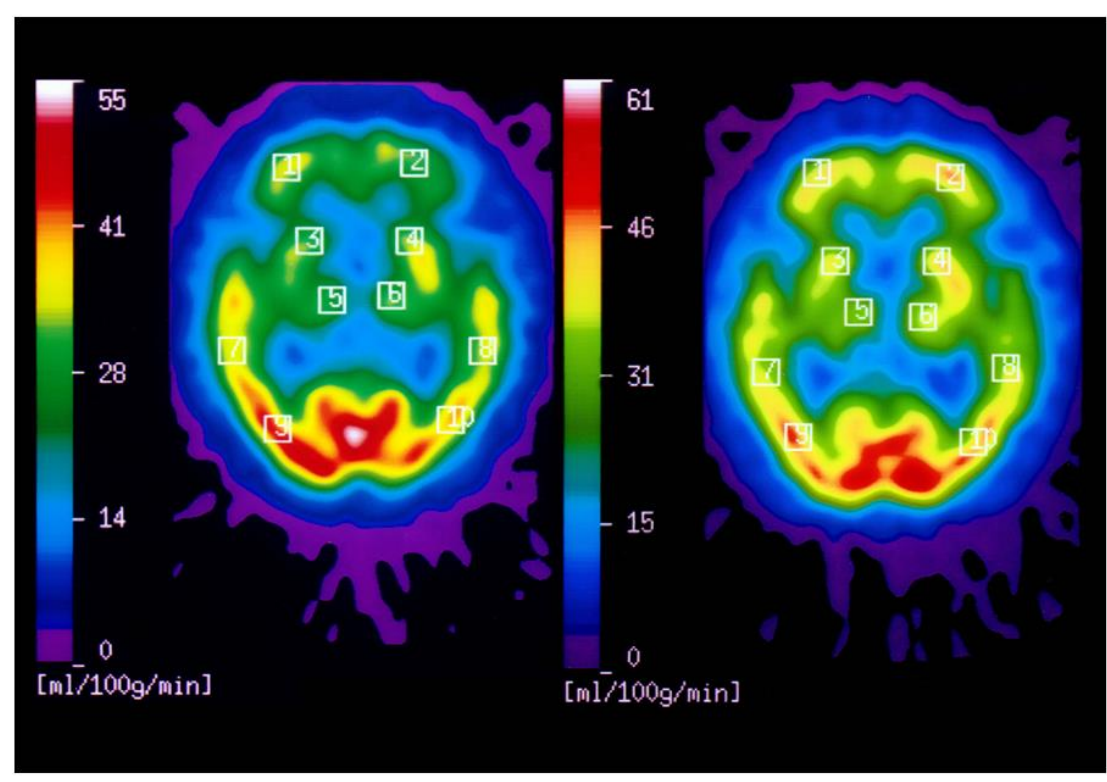

Figure 1 The changes in the cerebral blood flow at baseline (left) and the endpoint after two months of hachimijiogan treatment (right) were determined by performing single-photon emission computed tomography in a patient with severe Alzheimer's disease.

An increase in blood flow is evident in the bilateral anterior lobes (Figure 1). Ten patients with dementia showed an increase in cerebral blood flow, similar to that in the patient whose data is presented here.

\subsubsection{Efficacy of Hachimijiogan for Dementia}

In 2004, we published a paper titled, 'A Randomized, Double-Blind, Placebo-Controlled Clinical Trial of the Chinese Herbal Medicine "Ba Wei Di Huang Wan" in the Treatment of Dementia' [7]. Ba Wei Di Huang Wan is the Chinese term for hachimijiogan. In the study, 33 patients with dementia, who met the following criteria, were randomly assigned to the hachimijiogan treatment group ( $\mathrm{n}=$ $16)$ or placebo group ( $n=17): 1$ ) diagnosed with $A D$ or vascular dementia according to the Diagnostic and Statistical Manual of Mental Disorders, fourth edition (American Psychiatric Association); 2) mixed type of senile dementia [9]; 3) presence of dementia as indicated by a baseline Mini-Mental State Examination (MMSE) score of 0-25. 
The cognitive changes measured using the MMSE criterion in each group are presented in Figure 2 and based on the study conducted by Iwasaki et al. [7].

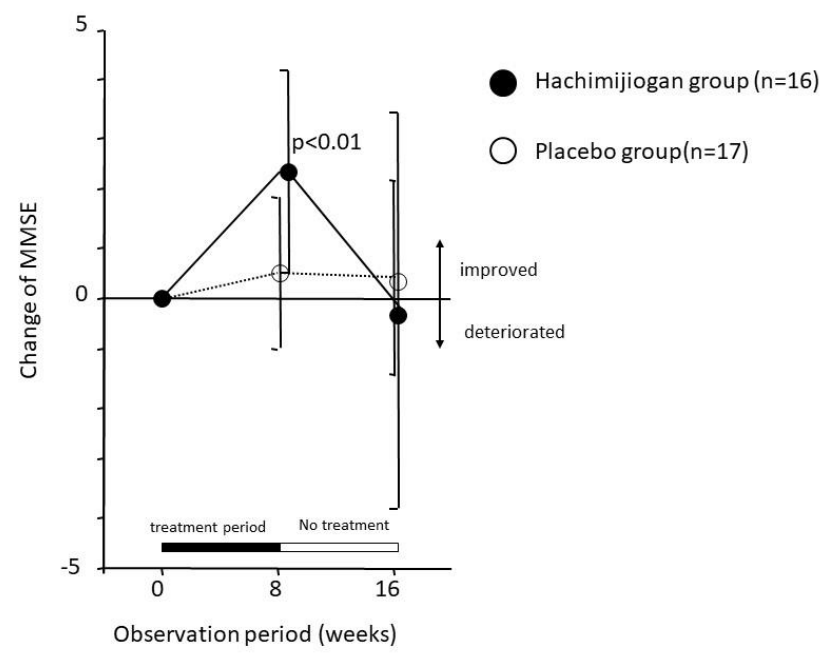

Figure 2 The change in the Mini-Mental State Examination scores of the hachimijiogan and placebo groups.

The MMSE score in the hachimijiogan group improved significantly at the end of drug administration compared to the baseline values (Tukey-Kramer post hoc test with repeated measures ANOVA, $P<0.01$ ). After eight weeks of treatment, the mean scores increased by 2.6 ( $95 \%$ confidence interval $[\mathrm{Cl}]:-4.1$ to 1.4$)$ and $0.6(95 \% \mathrm{Cl}:-2.0$ to 0.8 , no significance) in the hachimijiogan and placebo groups, respectively. The changes in the activities of daily living (ADL) measured using the Barthel Index [10] are shown in Figure 3 and based on the study conducted by Iwasaki et al. [7].

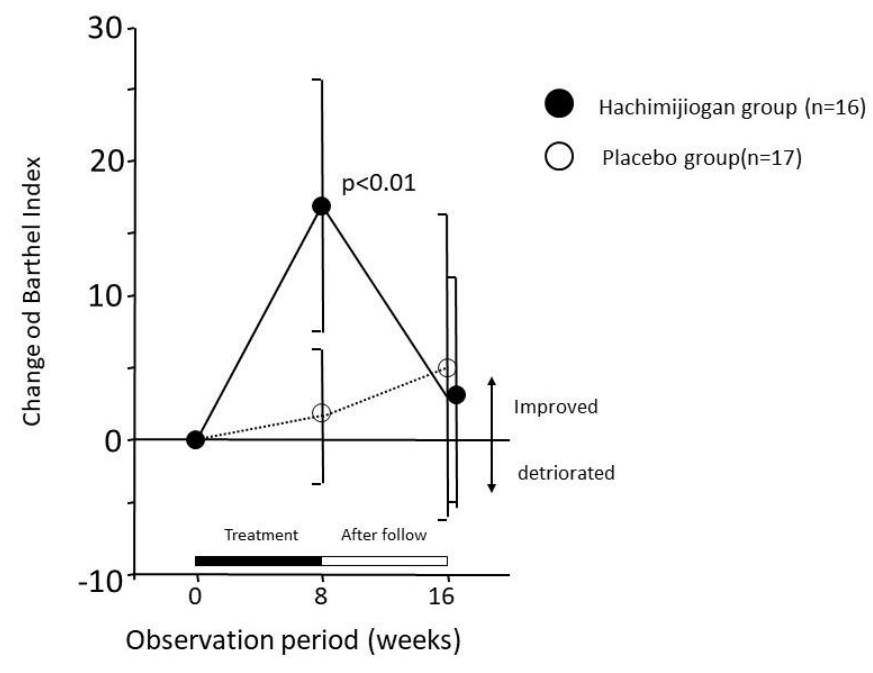

Figure 3 The changes in the activities of daily living were measured using the Barthel Index in the hachimijiogan and placebo groups. 
The Barthel Index improved significantly at the end of drug administration compared to the baseline value (Tukey-Kramer post hoc test, $P<0.01$ ). After eight weeks of treatment, the mean Barthel Index scores increased by $18.3(95 \% \mathrm{Cl}:-27.1$ to -9.5$)$ and $0.4(95 \% \mathrm{Cl}:-3.8$ to -3.1$)$ in the hachimijiogan and placebo groups, respectively. This small-scale study did not double-check the results and therefore could not confirm whether hachimijiogan was effective in the treatment of dementia. However, these results suggested the usefulness of Kampo medicine for dementia. The pharmacological effects of hachimijiogan were partially described in the study "Pharmacological action of hachimijiogan (Ba-wei-wan) on the metabolism of aged subjects" [11].

Currently, an exploratory, open-label standard treatment-controlled, randomized allocation, multicenter trial is underway to investigate the efficacy and safety of hachimijiogan in the treatment of mild $A D$ and is expected to further clarify the effect of hachimijiogan in dementia [12].

\subsection{Ninjin'yoeito (人参養栄湯) for Older People}

Takayama et al. conducted a review on the use of Ninjin'yoeito in modern medicine [13]. However, since only a few studies have been conducted on its effects that have only targeted the elderly, in this review, we discussed studies where the target population had a higher average age.

\subsubsection{Ninjin'yoeito for Alzheimer's Disease}

Kudoh et al. reported that Ninjin'yoeito maintained cognitive levels in AD patients [14] with a mean age of 74.5 years. This study conducted a two-year follow-up of patients treated with donepezil and Ninjin'yoeito and found improvements in cognitive function and reduction in ADrelated depression. Ohsawa et al. reported that Ninjin'yoeito improved anorexia and apathy and enhanced cognitive functions in patients with frailty and $A D$ (mean age of 82.6 years). Treatment with Ninjin'yoeito significantly reduced the score for "anorexia", which was based on the Neuropsychiatric Inventory (NPI) subcategory for eating disturbances, by week 4 (baseline: 4.85 \pm 0.58 ; 4 weeks: $3.06 \pm 0.60, p<0.05$; 8 weeks: $1.50 \pm 0.43, p<0.001 ; 12$ weeks: $1.00 \pm 0.44, p<0.001$ ) [15].

\subsubsection{Ninjin'yoeito for Chronic Respiratory Diseases}

Sadatani et al. reported that Ninjin'yoeito was effective in maintaining the nutritional status in elderly patients (between 72 and 75 years) with debilitating chronic respiratory diseases [16].

\subsubsection{Ninjin'yoeito for Cancer-Related Condition}

Fukuhara et al. found that Ninjin'yoeito alleviated the symptoms of fatigue in patients (average age of 71.9 years) undergoing chemotherapy for gastrointestinal cancer and reported that it might improve the prognosis after chemotherapy [17].

\subsection{Yokukansan (抑肝散) for Dementia}

\subsubsection{Effects of Yokukansan on Behavioral and Psychological Symptoms of Dementia}

Patients with dementia and their caregivers are not severely affected by cognitive deterioration. Instead, they are severely affected by delusions, hallucinations, anger, agitation, aggression, 
disinhibition, depression, and apathy, collectively known as BPSD. Antipsychotic agents are often used to treat BPSD; however, they can cause severe extrapyramidal symptoms due to falls and aspiration pneumonia. Based on an analysis of 17 placebo-controlled studies of four drugs used as antipsychotic agents, the United States Food and Drug Administration reported that the mortality rate for older patients with dementia was approximately 1.6-1.7 times that of patients in the placebo group [18].

Yokukansan (抑肝散) was developed by Xue Kai (薛鎧, the father) and Xue Ji (薛己, his son) during the Ming dynasty era [19]. However, Okamoto et al. [19] did not sufficiently describe yokukansan in their study. Koh Iwasaki, the corresponding author of this review, provided a more detailed description of yokukansan in the English version of Wikipedia in 2001 [20]. Although Wikipedia is irrelevant as a reference for scientific studies, we could not identify another adequate source of information on yokukansan in English.

In his textbook Hoeisatsuyo (保嬰撮要), Xue Kai (薛鎧) described this formulation as follows:

"This recipe improves convulsion, fever, gnash, restlessness, and enervation-induced fear. Moreover, it is effective against vomiting, feeling full, appetite loss, and stress-induced dysnystaxis."

Hoeisatsuyo (保嬰撮要) means “the essence of pediatrics." Therefore, Xue Kai (薛鎧) and Xue Ji (薛己) might have mainly used yokukansan to treat children. In 2005, Iwasaki et al. conducted a randomized controlled study on the efficacy of yokukansan for treating BPSD in patients with dementia, including hallucinations, delusions, and anger [21]. Yokukansan contains Atractylodis Lanceae Rhizoma, Poria, Cnidii Rhizoma, Uncariae Uncis Cum Ramulus, Angelicae Acutilobae Radix, Bupleuri Radix, and Glycyrrhizae Radix. BPSD was examined using the NPI, with lower scores indicating better conditions [22]. The changes in BPSD reflected by the NPI score in each group are shown in Figure 4, based on the study conducted by Iwasaki et al. [21]. There was a significant improvement in the NPI score in the yokukansan group ( $n=27$, from $37.9 \pm 16.1$ to $19.5 \pm 15.6$ [mean \pm standard deviation], $P<0.001)$ but not in the control group $(n=25)$. The changes in the ADL are shown in Figure 5, based on the study conducted by Iwasaki et al. [21].

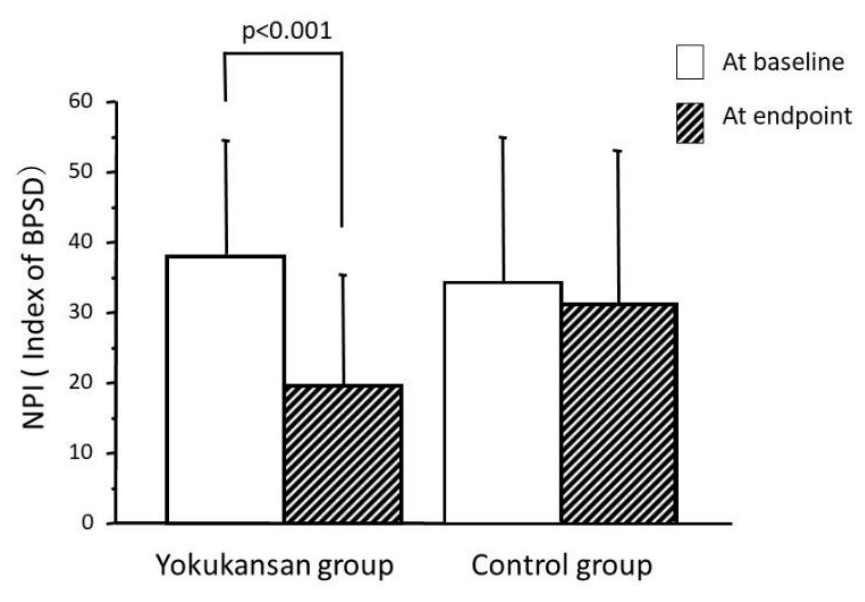

Figure 4 A change in the Neuropsychiatric Inventory score in the yokukansan and control groups. 


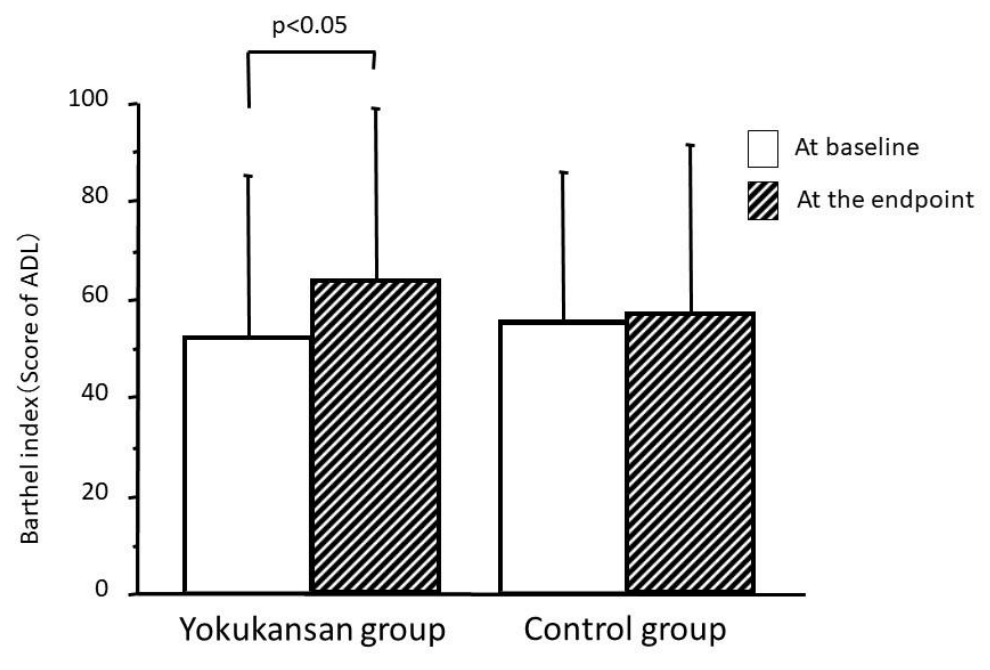

Figure 5 The changes in the activities of daily living were measured using the Barthel Index in the yokukansan and control groups.

ADL measured using Barthel index scores improved significantly in the yokukansan group (from $56.4 \pm 34.2$ to $62.9 \pm 35.2, P<0.05$ ) but not in the control group. Generally, antipsychotic agents do not improve $A D L$, owing to their adverse effects. A systematic review and meta-analysis validated the efficacy of yokukansan for BPSD [23, 24]. However, yokukansan improves "positive symptoms," including delusions, hallucinations, anger, agitation, aggression, and disinhibition, but not "negative symptoms," such as depression and apathy. The corresponding author, Koh Iwasaki, treated a patient with $A D$ who was prescribed yokukansan by her physician, although she presented only "negative symptoms", such as depression, apathy, and appetite loss. Finally, she was unable to eat or drink and was treated in our emergency clinic for severe dehydration and malnutrition. Moreover, yokukansan contains Glycyrrhizae Radix, which can cause hypokalemia. Therefore, yokukansan should not be used for more than one month, and serum potassium levels should be checked after one month of use.

\subsubsection{Yokukansan for Visual Hallucinations in Dementia with Lewy Bodies}

In a study on patients suffering from dementia with Lewy bodies (DLB) who were having visual hallucinations, treatment with yokukansan ameliorated visual hallucinations in some patients with DLB [21]. Subsequently, a case series reported the effects of yokukansan on visual hallucinations in patients with DLB [25]. The changes in the NPI subscale score in patients with DLB are shown in Figure 6, based on the study conducted by Iwasaki et al. [25]. 


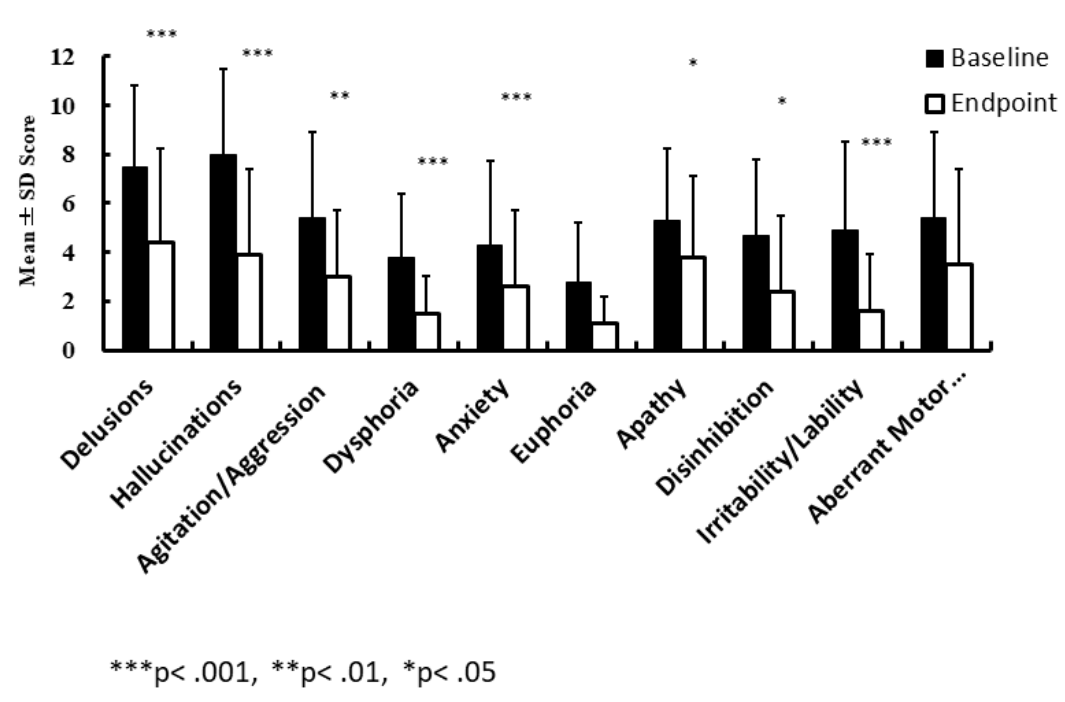

Figure 6 The changes in the Neuropsychiatric Inventory subscale scores in the yokukansan group between baseline and endpoint.

There was a significant improvement in delusion, hallucinations, agitation, dysphoria, anxiety, and irritability experienced by patients with DLB (Figure 6). Although significant improvements were also observed in apathy and irritability, they were not starkly evident. The change in the caregiver burden measured using the Zarit Burden Interview-Japanese edition [26] is shown in Figure 7 and is based on the study by Arai et al. [26]. The total score in the Zarit Burden Interview decreased in all 51 patients after four weeks of treatment with yokukansan (Figure 7A). Of these, 22 patients who provided consent to be examined for changes in the Zarit Burden Interview score showed a significant decrease in the score after two weeks (Figure 7B), which indicated that the effects of yokukansan can be seen after treatment for a short while (two weeks). 

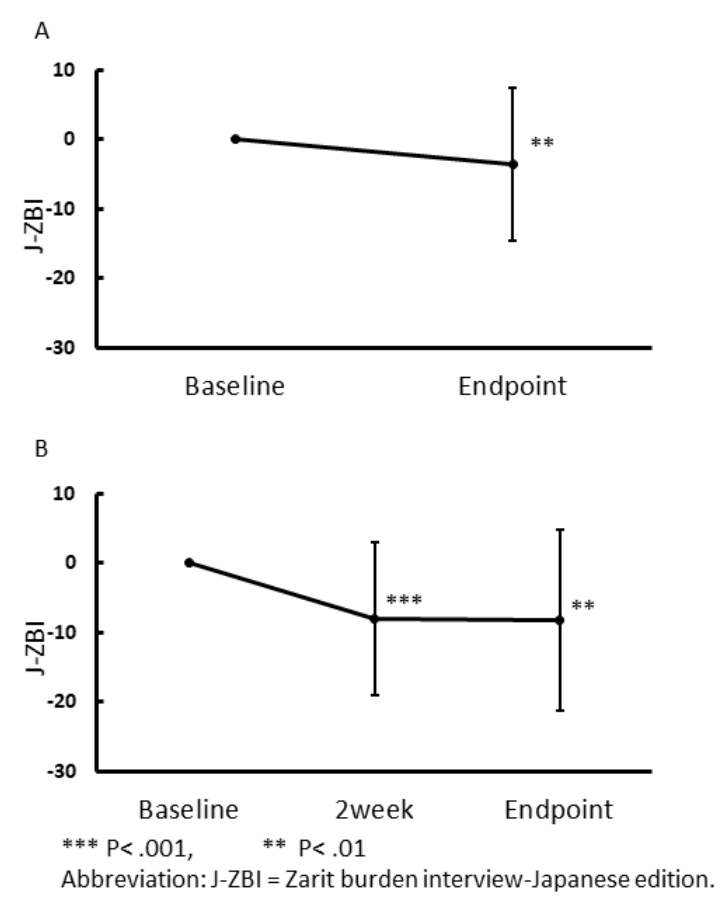

Figure 7 The changes in the total scores of the Zarit Burden Interview-Japanese edition (J-ZBI) in patients with dementia with Lewy bodies.

(A) The participants $(n=51)$ were evaluated using the J-ZBI, which was administered twice, at baseline and after four weeks. (B) Some of the patients $(n=22)$ agreed to undergo assessment three times (weeks 0, 2, and 4).

Yokukansan treatment significantly decreased caregiver burden. Four patients had potassium levels $<3.5 \mathrm{mEq} / \mathrm{L}$ at the end of the study. One patient showed spasticity and BPSD worsening, which recovered after discontinuing yokukansan. Another patient showed exacerbation of hypotension. A few patients experienced adverse events, including edema, gastrointestinal dysfunction, spasticity, and exacerbation of delusions and hallucinations. No cognitive changes were observed during the study period [25].

\subsection{Role of Hangekobokuto (半夏厚朴湯) in Preventing Aspiration Pneumonia}

Among older people, aspiration pneumonia is a major cause of hospitalization and death [27]. Risk factors for aspiration pneumonia include sputum suctioning, deterioration of swallowing function in the past three months, dehydration, and dementia [28]. Figure 8 shows a radiograph of a patient with aspiration pneumonia in the right lung (right) and the corresponding brain computed tomography image (left) (the arrows indicate the areas with ischemia-induced damage in the bilateral basal ganglia). Ischemic damage in the basal ganglia can cause aspiration pneumonia [29]. 

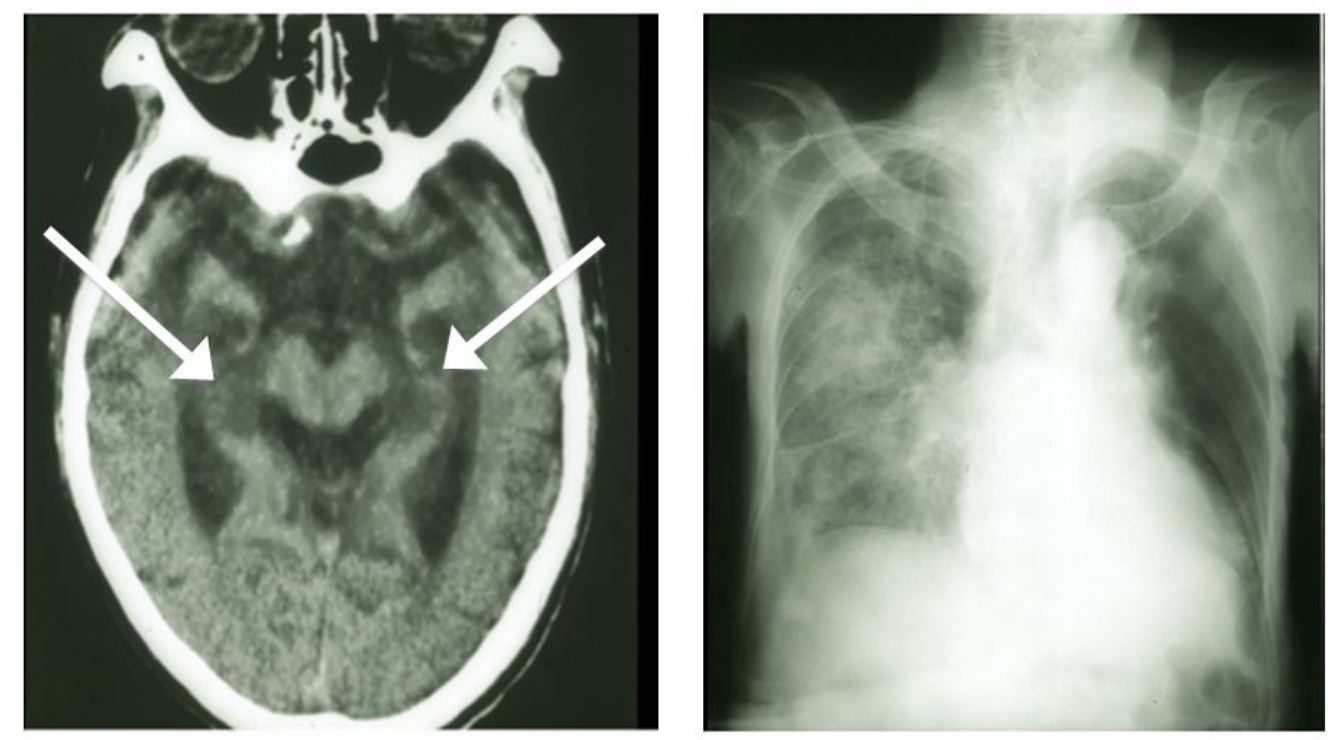

Figure 8 Aspiration pneumonia.

A radiograph depicting aspiration pneumonia in the right lung (right) with the corresponding brain computed tomography scan (left). The arrows indicate the areas with ischemia-induced damage in the bilateral basal ganglia.

Hangekobokuto (半夏厚朴湯) is among the medicines described in Kinkiyoryaku (金蕢要略) [4]. It comprises five herbs, including Pinelliae Tuber, Poria, Magnoliae Cortex, Perillae Herba, and Zingiberis Rhizoma. Hangekobokuto significantly improves swallowing [30] and cough reflexes [31]. A prospective, observer-blinded, randomized control trial was conducted to determine whether hangekobokuto could improve pneumonia in frail old individuals [32]. The study assessed 104 older patients (31 men and 73 women; mean age \pm standard deviation: $83.5 \pm 7.8$ years) with stroke, AD, and/or Parkinson's disease, who were admitted to two long-term care hospitals for handicapped older patients in Japan. The occurrence of pneumonia, mortality due to pneumonia, and the amount of daily self-feeding were measured. Ninety-five participants (mean age, 84.0 years; male: female, 28:67) were randomly assigned to the hangekobokuto treatment group ( $n=47$ ) or control group ( $n$ $=48$ ) and were administered hangekobokuto or placebo, respectively, for 12 months.

As shown in Figure 9, hangekobokuto significantly reduced the incidence of pneumonia; the figure is from the study conducted by Iwasaki et al. [32]. Pneumonia occurred in 4 (9.1\%) and 14 (29.2\%) patients in the hangekobokuto and control groups, respectively. A significant betweengroup difference was observed in the frequency of the occurrence of pneumonia $(P=0.008)$. Moreover, hangekobokuto reduced pneumonia-related mortality (Figure 10 was taken from the study conducted by Iwasaki et al. [32]) and preserved the amount of daily self-feeding (Figure 11). 


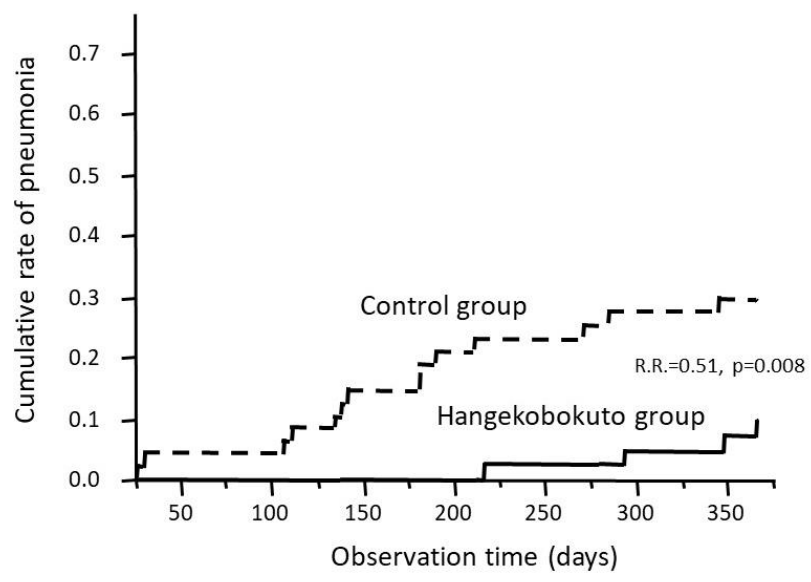

Figure 9 The accumulated rate of pneumonia onset in the hangekobokuto and control groups.

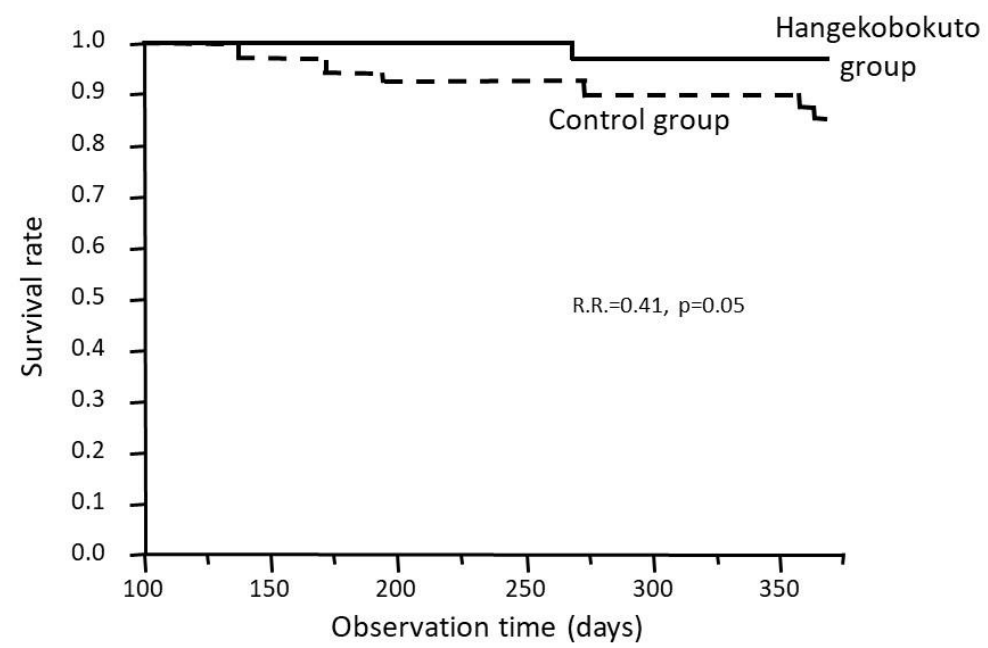

Figure 10 The accumulated survival rate in the hangekobokuto and control groups.

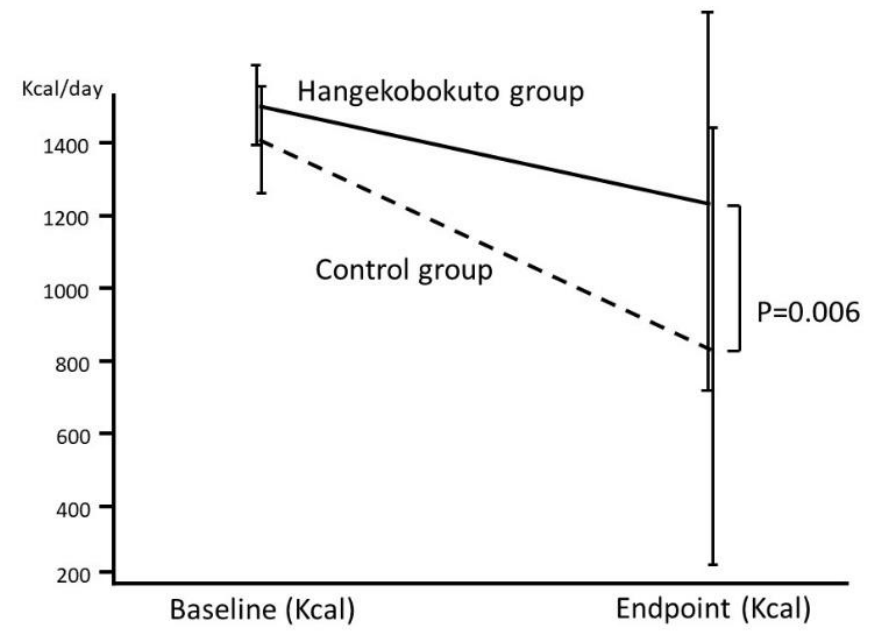

Figure $\mathbf{1 1}$ The change in the daily amount of self-feeding in the hangekobokuto and control groups. 
As shown in Figure 11, the amount of daily self-feeding decreased from 1,443.2 $\pm 105.3 \mathrm{Kcal}$ (mean \pm standard deviation) to $1,250.0 \pm 511.5 \mathrm{Kcal}$ in the hangekobokuto group and from 1,401.5 $\pm 174.1 \mathrm{Kcal}$ to $844.0 \pm 636.9 \mathrm{Kcal}$ in the control group; the differences between the two groups were significant $(P=0.006)$.

Recently, another double-blinded randomized control study demonstrated the effect of hangekobokuto in reducing aspiration pneumonia [33]. In this study conducted with patients undergoing cardiovascular surgery, the proportion of subjects with postoperative aspiration pneumonia was significantly lower in the hangekobokuto group than in the placebo group $(P=$ 0.017). In high-risk patients suffering from aspiration pneumonia, the proportion was significantly lower in the hangekobokuto group than in the placebo group $(P=0.015)$. The proportion of subjects with swallowing disorders tended to be lower in the hangekobokuto group than in the placebo group $(P=0.091)$, and in high-risk patients, the proportion was significantly lower in the hangekobokuto group than in the placebo group $(P=0.038)$.

\subsection{Recovery of Oral Feeding in Older Japanese Individuals After Long-term Tube Feeding}

In Japan, long-term tube feeding is used to manage patients with stroke or appetite loss who cannot ingest food orally. The two most commonly used methods of tube feeding are nasogastric tube (NGT) insertion and percutaneous endoscopic gastrostomy (PEG) [34]. Although tube feeding facilitates nutritional management, it causes distress and inconvenience to patients, especially older people with dementia; moreover, it significantly reduces the quality of life [35-37]. Generally, older patients with long-term enteral feeding via NGT insertion or PEG require permanent tube feeding. We reviewed the medical records of patients who attempted to resume oral feeding after receiving NGT or PEG treatment at Miyama Hospital for $\geq 12$ months. Hangekobokuto was prescribed to patients with a swallowing reflex time $>4 \mathrm{~s}$. Half of the patients (7/14) managed to resume oral feeding [38]; furthermore, hangekobokuto significantly improved the swallowing reflex (Figure 12, taken from the study conducted by Nogami et al. [38]). This suggested that some older patients with long-term enteral feeding can stop tube feeding and that hangekobokuto might provide support during withdrawal.

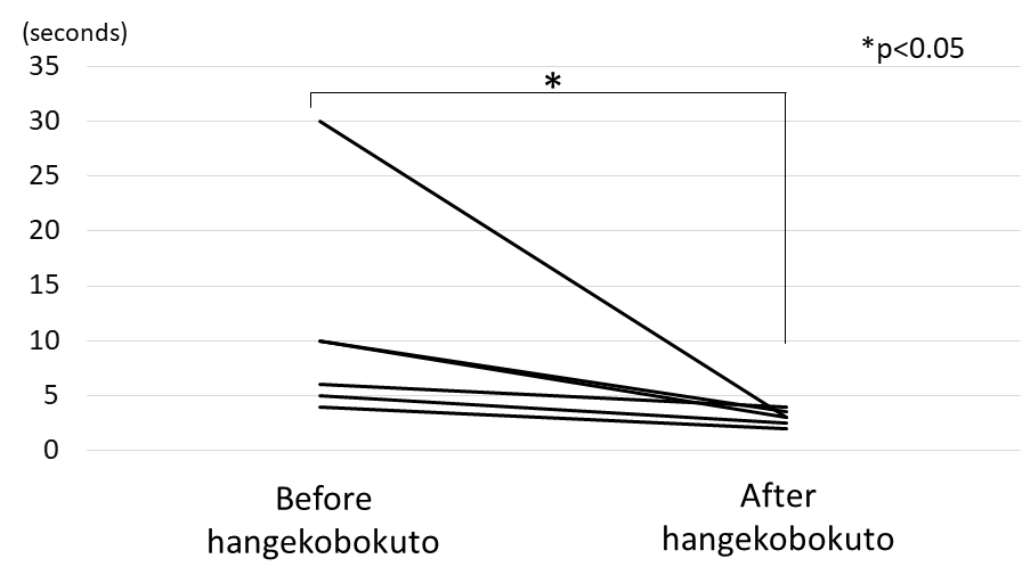

Figure 12 The changes in the swallowing reflex time before and after hangekobokuto administration in seven patients who received tube feeding. 


\subsection{Kampo Medicines for Gastroesophageal Regurgitation}

Gastroesophageal regurgitation is an important cause of aspiration pneumonia [39]. Rikkunshito (六君子湯) improves acid reflux in post-gastrectomy patients [40]. The administration of rikkunshito also improves acid-related dysmotility scores [41]. Kato et al. reported that hangekobokuto significantly improved the respiratory symptoms in reflux esophagitis [42]. Thus, these Kampo prescriptions might prevent gastroesophageal regurgitation and aspiration pneumonia.

\subsection{Constipation}

Many elderly individuals experience constipation. Since ancient times, Sennae Folium in Europe, Rhei Rhizoma in Oriental countries, and magnesium oxide have been used as purgatives. However, patients with chronic constipation develop resistance to Sennae Folium, given that its common active components are sennosides. Although magnesium oxide does not cause resistance, it is quite potent for older people and increases the risk of hypermagnesemia.

\subsubsection{Mashiningan (麻子仁丸)}

Mashiningan (麻子仁丸) is a traditional purgative that has been mentioned in Shokanron (傷寒 論) [43] and Kinkiyoryaku (金貴要略) [4], both of which were written by Zhang Zhongjing (張仲景) in the second century. Mashiningan contains six herbs, including Cannabis Fructus, Rhei Rhizoma, Aurantii Fructus Immaturus, Armeniacae Semen, Magnoliae Cortex, and Paeoniae Radix. A doubleblinded randomized control study conducted in 2011 [44] and a meta-analysis [45] confirmed the efficacy of mashiningan as a purgative. Mashiningan is often used by older people since a single dose contains only $1.3 \mathrm{~g}$ of Rhei Rhizoma extract, which renders it suitable for frail old individuals.

\subsubsection{Daikenchuto (大建中湯)}

Patients with stroke often experience constipation [46]. A previous study showed that constipation in stroke survivors improved after the administration of daikenchuto (大建中湯), which was prescribed in Kinkiyoryaku (金貴要略) and contains Ginseng Radix, Zingiberis Rhizoma Processum, Zanthoxyli Piperiti Pericarpium, and Koi (candy made from maltose) [47]. The clinical scores for constipation (CSS) [48] in stroke survivors are shown in Figure 13; the scores improved following daikenchuto treatment [47]. 


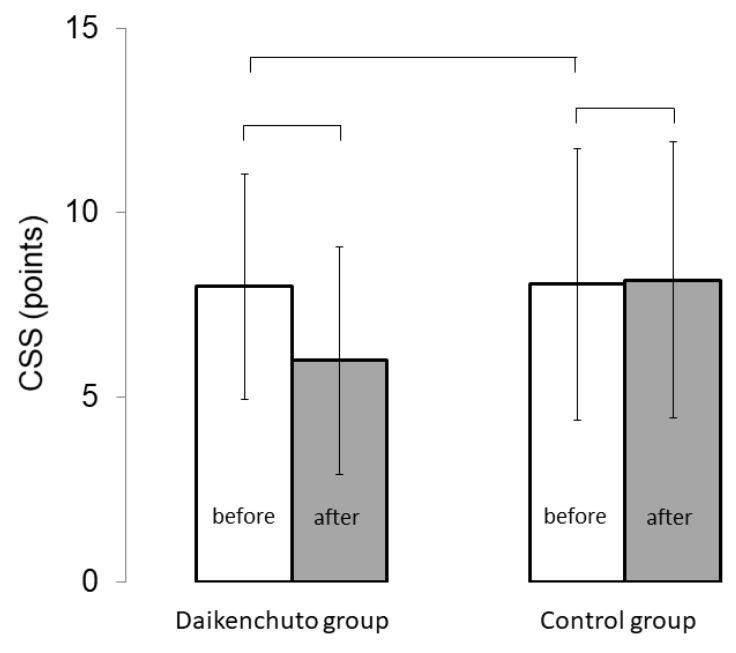

Figure 13 The changes in the clinical constipation scores (CSSs) of the daikenchuto and control groups.

In the daikenchuto group, the CSS significantly improved from $8.0 \pm 3.1$ to $6.0 \pm 3.1$ points (paired t-test, $P<0.01)$. The correlation between age and the changes in the CSS for participants in the daikenchuto group was not significant $(n=17)(r=-0.16, P=0.53)$. The control group did not show any significant improvement. Daikenchuto is often used to prevent ileus after colectomy. The details are available in the review by Takayama et al. [49].

\section{Acupuncture}

Acupuncture is a part of Kampo medicine. Suzuki et al. determined the efficacy of acupuncture for improving the nutritional status of patients with chronic obstructive pulmonary disease [50]. Weight loss is a prominent manifestation of obstructive pulmonary disease. In the study by Suzuki et al., the change in body weight after 12 weeks was significantly greater in the real acupuncture group (RAG) compared to the placebo acupuncture group (PAG) (mean [standard deviation] difference from baseline: 2.5 [0.4] in RAG and -0.5 [1.4] in PAG; mean difference between the groups: $3.00,95 \% \mathrm{Cl}, 2.00$ to 4.00 with the analysis of covariance). Patients in the RAG group also showed improvements in the results of the nutritional hematological examination (retinol-binding protein, prealbumin, transferrin, and hemoglobin), inflammatory biomarkers (tumor necrosis factor- $\alpha$, interleukin 6, serum amyloid A, high-sensitivity C-reactive protein, and carboxyhemoglobin), and the BODE index. Kikuchi et al. investigated the efficacy of acupuncture using push needles to improve the swallowing reflex in older people with cerebrovascular diseases. The study recruited 29 individuals (10 men, 19 women; mean age \pm standard deviation, $82.2 \pm 7.1$ ) and found that the ST36 and KI3 press needles (Pyonex; Seirin Corporation, Shizuoka, Japan) significantly reduced the latency of the swallowing reflex ( $6.9 \pm 2.3 \mathrm{~s}$ vs. $2.5 \pm 0.3 \mathrm{~s}, P=0.005$ ) [51].

\section{Background Philosophy}

Kampo and TCM are used in the field of geriatrics. However, it is important to understand not only the data and efficacies but also the background philosophy and properties of these traditional 
prescriptions. For example, shakuyakukanzoto (苟薬甘草湯) provides immediate relief from cramps, but most Japanese doctors do not know that shakuyakukanzoto contains $6 \mathrm{~g}$ of Glycyrrhizae Radix. Therefore, they administer shakuyakukanzoto continuously for many months, which is an incorrect method in Kampo medicine and causes pseudoaldosteronism and hypokalemia. According to classical literature, the world is composed of Qi (気), which refers to the existence of a function without form. Moreover, all forms of life are parts of Qi that are only formed temporarily.

Geriatrics and gerontology involve the treatment and prevention of aging and death. From the perspective of classical TCM, every creature is a part of the circulating Qi, and no individual can distinguish itself from the objective world. The difference between self and non-self is vague. For example, mitochondria are present in all human cells. However, mitochondria have biological membranes and DNA, suggesting that they were independent organisms long ago. Therefore, it is unclear whether mitochondria are a part of the human cell. Moreover, the difference between life and death is vague. For example, HeLa cells were obtained from a woman called Henrietta Leanne Lacks [52]. Although she died in 1951, the HeLa cell line remains alive. Therefore, there is no definite demarcation between the self and non-self or between life and death. TCM partly borrowed these philosophies from Buddhism. Living things, including humans, are only temporary manifestations of $Q_{i}$ in the world. Buddhism postulates that all living things should age, fall sick, and die, without exception [53]. This is also the fundamental concept of classical TCM gerontology. In Section 3.6, we discussed the recovery of oral feeding in elderly Japanese people after long-term tube feeding. The condition of elderly patients supported by several tubes at the last stage of life is called the Spaghetti syndrome in Japan. Kouteidaikei (黄帝内経), the ancient treatise described in Section 1, mentioned that if a person cannot eat, that person shall die; however, the Spaghetti syndrome represents the antithesis to this philosophy. Although the Spaghetti syndrome might extend the life span of a person, elderly individuals might not want such treatment forms at the last stage of life. Thus, the fundamental concepts of classical TCM gerontology should be understood in greater detail.

\section{Acknowledgments}

We would like to thank Editage (www.editage.jp) for English language editing.

\section{Author Contributions}

TN, MA, and KI prepared, edited, and reviewed the manuscript. The final manuscript was read and approved by all authors. Furthermore, all authors meet the requirements of authorship, and each author believes that the manuscript represents honest work.

\section{Funding}

The study did not receive funding from any external sources.

\section{Competing Interests}

T. Nogami and M. Arai received lecture fees from Tsumura \& Co, and their affiliation gained a research grant from Tsumura $\&$ Co., all of which are unrelated to this review. 


\section{References}

1. The Japanese pharmacopoeia 17th edition. The MHLW Ministerial Notification No. 64, March 7, 2016 [Internet]. Tokyo: Ministry of Health, Labour and Welfare of Japan; 2016 [cited date 2021 June 5]. Available from: https://www.mhlw.go.jp/file/06-Seisakujouhou-11120000lyakushokuhinkyoku/JP17 REV 1.pdf.

2. Cesari M, Marzetti E, Canevelli M, Guaraldi G. Geriatric syndromes: How to treat. Virulence. 2017; 8: 577-585.

3. Takayama S, Iwasaki K. Systematic review of traditional Chinese medicine for geriatrics. Geriatr Gerontol Int. 2017; 17: 679-688.

4. New World Encyclopedia. Huangdi Neijing [Internet]. Saint Paul: Paragon House Publishers; 2018 [cited date 2022 January 12]. Available from:

https://www.newworldencyclopedia.org/entry/Huangdi_Neijing.

5. Wiseman N, Lue WS. Essential prescriptions of the golden cabinet-translation \& commentaries. Taos: Paradigm Publications; 2012.

6. Marsden S. Ba Wei Di Huang Wan (Rehmannia eight). In: Dr. Steve Marsden's essential guide to Chinese herbal formulas-bridging science and tradition in integrative veterinary medicine. Yeppoon: College of Integrative Veterinary Therapies; 2014. pp.55-59.

7. Iwasaki K, Kobayashi S, Chimura Y, Taguchi M, Inoue K, Cho S, et al. A randomized, double-blind, placebo-controlled clinical trial of the Chinese herbal medicine "ba wei di huang wan" in the treatment of dementia. J Am Geriatr Soc. 2004; 52: 1518-1521.

8. Iwasaki K, Kobayashi S, Chimura Y, Taguchi M, Inoue K, Akiba T, et al. Effects of the Chinese herbal medicine 'Ba Wei Di Huang Wan' in the treatment of dementia: A SPECT cerebral blood flow examination and a randomized, double-blind, placebo-controlled clinical trial for cognitive function and ADL. Geriatr Gerontol Int. 2004; 4: S124-S128.

9. Kalaria R. Similarities between Alzheimer's disease and vascular dementia. J Neurol Sci. 2002; 203: 29-34.

10. Mahoney Fl. Functional evaluation: The Barthel index. Md State Med J. 1965; 14: 61-65.

11. Haranaka R, Okada N, Kosoto H, Ohwada S, Kobayashi M, Yoshida H. Pharmacological action of hachimijiogan (Ba-wei-wan) on the metabolism of aged subjects. Am J Chinese Med. 1986; 14: 59-67.

12. Kainuma M, Funakoshi K, Ouma S, Yamashita KI, Ohara T, Yoshiiwa A, et al. The efficacy and safety of hachimijiogan for mild Alzheimer disease in an exploratory, open standard treatment controlled, randomized allocation, multicenter trial: A study protocol. Medicine. 2020; 99: e22370.

13. Takayama S, Arita R, Ohsawa M, Kikuchi A, Yasui H, Makino T, et al. Perspectives on the use of Ninjin'yoeito in modern medicine: A review of randomized controlled trials. Evid Based Complementary Altern Med. 2019; 2019: 9590260.

14. Kudoh C, Arita R, Honda M, Kishi T, Komatsu Y, Asou H, et al. Effect of ninjin'yoeito, a Kampo (traditional Japanese) medicine, on cognitive impairment and depression in patients with Alzheimer's Disease: 2 years of observation. Psychogeriatrics. 2016; 16: 85-92.

15. Ohsawa M, Tanaka Y, Ehara Y, Makita S, Onaka K. A possibility of simultaneous treatment with the multicomponent drug, Ninjin'yoeito, for anorexia, apathy, and cognitive dysfunction in frail Alzheimer's disease patients: An open-label pilot study. J Alzheimers Dis Rep. 2017; 1: 229-235. 
16. Sasatani Y, Okauchi S, Ohara G, Kagohashi K, Satoh H. Long-term maintenance of nutritional status with ninjinyoueito in terminal patients with chronic respiratory disease: Two case reports. Biomed Rep. 2020; 12: 121-124.

17. Fukuhara K, Edagawa E, Nishioka T, Shinjo S. The effect of Ninjinyoeito for patients undergoing chemotherapy. Gan to Kagaku ryoho. Cancer Chemother. 2019; 46: 1033-1037.

18. Cruzan S. FDA Issues public health advisory for antipsychotic drugs used for treatment of behavioral disorders in elderly patients [Internet]. Silver Spring: Food and Drug Administration; 2005 [cited date 2021 May 5th] Available from: http://psychrights.org/drugs/ANS01350.html.

19. Okamoto H, lyo M, Ueda K, Han C, Hirasaki Y, Namiki T. Yokukan-san: A review of the evidence for use of this Kampo herbal formula in dementia and psychiatric conditions. Neuropsychiatr Dis Treat. 2014; 10: 1727-1742.

20. Wikipedia.org. Yokukansan [Internet]. Saint Petersburg: The Wikipedia Foundation; 2021 [cited date 2022 January 12]. Available from: https://en.wikipedia.org/wiki/Yokukansan.

21. Iwasaki K, Satoh-Nakagawa T, Maruyama M, Monma $Y$, Nemoto M, Tomita N, et al. A randomized, observer-blind, controlled trial of the traditional Chinese medicine Yi-Gan San for improvement of behavioral and psychological symptoms and activities of daily living in dementia patients. J Clin Psychiatry. 2005; 66: 16809.

22. Cummings JL, Mega M, Gray K, Rosenberg-Thompson S, Carusi DA, Gornbein J. The neuropsychiatric inventory: Comprehensive assessment of psychopathology in dementia. Neurology. 1994; 44: 2308-2314.

23. Matsuda Y, Kishi T, Shibayama H, Iwata N. Yokukansan in the treatment of behavioral and psychological symptoms of dementia: A systematic review and meta-analysis of randomized controlled trials. Hum Psychopharmacol. 2013; 28: 80-86.

24. Matsunaga S, Kishi T, Iwata N. Yokukansan in the treatment of behavioral and psychological symptoms of dementia: An updated meta-analysis of randomized controlled trials. J Alzheimers Dis. 2016; 54: 635-643.

25. Iwasaki K, Kosaka K, Mori H, Okitsu R, Furukawa K, Manabe Y, et al. Improvement in delusions and hallucinations in patients with dementia with Lewy bodies upon administration of yokukansan, a traditional Japanese medicine. Psychogeriatrics. 2012; 12: 235-241.

26. Arai $\mathrm{Y}, \mathrm{MD} \mathrm{KK}$, Hosokawa $\mathrm{T}$, Washio M, Miura H, Hisamichi S. Reliability and validity of the Japanese version of the Zarit Caregiver Burden Interview. Psychiatry Clin Neurosci. 1997; 51: 281-287.

27. Kaplan V, Angus DC, Griffin MF, Clermont G, Scott Watson R, Linde-Zwirble WT. Hospitalized community-acquired pneumonia in the elderly: Age-and sex-related patterns of care and outcome in the United States. Am J Respir Crit Care Med. 2002; 165: 766-772.

28. Manabe T, Teramoto S, Tamiya N, Okochi J, Hizawa N. Risk factors for aspiration pneumonia in older adults. PloS One. 2015; 10: e0140060.

29. Nakagawa T, Arai H, Kikuchi R, Manabe K, Sasaki H. High incidence of pneumonia in elderly patients with basal ganglia infarction. Arch Intern Med. 1997; 157: 321-324.

30. Iwasaki K, Wang Q, Nakagawa T, Suzuki T, Sasaki H. The traditional Chinese medicine banxia houpo tang improves swallowing reflex. Phytomedicine. 1999; 6: 103-106.

31. Iwasaki K, Cyong JC, Kitada S, Kitamura H, Ozeki Jl, Satoh Y, et al. A traditional Chinese herbal medicine, banxia houpo tang, improves cough reflex of patients with aspiration pneumonia. J Am Geriatr Soc. 2002; 50: 1751-1752. 
32. Iwasaki K, Kato S, Monma Y, Niu K, Ohrui T, Okitsu R, et al. A pilot study of banxia houpu tang, a traditional Chinese medicine, for reducing pneumonia risk in older adults with dementia. J Am Geriatr Soc. 2007; 55: 2035-2340.

33. Kawago K, Nishibe T, Shindo S, Inoue H, Motohashi S, Akasaka J, et al. A double-blind randomized controlled trial to determine the preventive effect of hangekobokuto on aspiration pneumonia in patients undergoing cardiovascular surgery. Ann Thorac Cardiovasc Surg. 2019; 25: 318-325.

34. Ogita $\mathrm{M}$, Utsunomiya $\mathrm{H}$, Akishita $\mathrm{M}$, Arai $\mathrm{H}$. Indications and practice for tube feeding in Japanese geriatricians: Implications of multidisciplinary team approach. Geriatr Gerontol Int. 2012; 12: 643-651.

35. Iwasaki K, Kurachi M, Nogami T, Takayama S. Traditional Chinese therapy initiates oral feeding in a stroked woman after three years of nasogastric tube feeding. J Fam Med Prim Care. 2019; 8: 3059-3060.

36. Messing B. Long-term outcome and quality of life of adult patients on home parenteral nutrition. Clin Nutr. 1995; 14: 24-27.

37. Winkler MF. Quality of life in adult home parenteral nutrition patients. J Parenter Enteral Nutr. 2005; 29: 162-170.

38. Nogami T, Kurachi M, Hukushi T, Iwasaki K. Recovery of oral feeding in Japanese elderly people after long-term tube feeding: A challenge in Miyama Hospital. J Fam Med Prim Care. 2020; 9: 3977-3980.

39. Virkkunen I, Ryynänen S, Kujala S, Vuori A, Piilonen A, Kääriä JP, et al. Incidence of regurgitation and pulmonary aspiration of gastric contents in survivors from out-of-hospital cardiac arrest. Acta Anaesthesiol Scand. 2007; 51: 202-205.

40. Gunji S, Ueda S, Yoshida M, Kanai M, Terajima H, Takabayashi A. Effects of rikkunshito, a kampo medicine, on quality of life after proximal gastrectomy. J Surg Res. 2013; 185: 575-580.

41. Sakata Y, Tominaga K, Kato M, Takeda H, Shimoyama Y, Takeuchi T, et al. Clinical characteristics of elderly patients with proton pump inhibitor-refractory non-erosive reflux disease from the G-PRIDE study who responded to rikkunshito. BMC Gastroenterol. 2014; 14: 116.

42. Kato S, Nakajima T, Matsuda T. Efficacy of hangekobokuto for respiratory symptom related with reflex esophagitis. Kampo Newest Ther. 2005; 14: 333-338.

43. Zhang Z. Shang Han Lun on cold damage: Translation \& commentaries. 1st ed. Brookline: Paradigm Publications; 1998.

44. Cheng CW, Bian ZX, Zhu LX, Wu JC, Sung JJ. Efficacy of a Chinese herbal proprietary medicine (Hemp Seed Pill) for functional constipation. Am J Gastroenterol. 2011; 106: 120-129.

45. Yang $M$, Feng $Y$, Zhang $Y L$, Smith $C M$, Hou $Y N$, Wang $H$, et al. Herbal formula MaZiRenWan (Hemp Seed Pill) for constipation: A systematic review with meta-analysis. Phytomedicine. 2021; 82: 153459.

46. Su Y, Zhang X, Zeng J, Pei Z, Cheung RT, Zhou QP, et al. New-onset constipation at acute stage after first stroke: Incidence, risk factors, and impact on the stroke outcome. Stroke. 2009; 40: 1304-1309.

47. Numata $T$, Takayama S, Tobita M, Ishida S, Katayose $D$, Shinkawa $M$, et al. Traditional Japanese medicine daikenchuto improves functional constipation in poststroke patients. Evid Based Complementary Altern Med. 2014; 2014: 231258. 
48. Agachan F, Chen T, Pfeifer J, Reissman P, Wexner SD. A constipation scoring system to simplify evaluation and management of constipated patients. Dis Colon Rectum. 1996; 39: 681-685.

49. Takayama S, Tomita N, Arita R, Ono R, Kikuchi A, Ishii T. Kampo Medicine for various agingrelated symptoms: A review of geriatric syndrome. Front Nutr. 2020; 7: 86.

50. Suzuki M, Muro S, Fukui M, Ishizaki N, Sato S, Shiota T, et al. Effects of acupuncture on nutritional state of patients with stable chronic obstructive pulmonary disease (COPD): Reanalysis of COPD acupuncture trial, a randomized controlled trial. BMC Complement Altern Med. 2018; 18: 287.

51. Kikuchi A, Seki T, Takayama S, Iwasaki K, Ishizuka S, Yaegashi N. Effect of press needles on swallowing reflex in older adults with cerebrovascular disease: A randomized double-blind controlled trial. J Am Geriatr Soc. 2014; 62: 2438-2440.

52. Scherer WF, Syverton JT, Gey GO. Studies on the propagation in vitro of poliomyelitis viruses. IV. Viral multiplication in a stable strain of human malignant epithelial cells (strain HeLa) derived from an epidermoid carcinoma of the cervix. J Exp Med. 1953; 97: 695-710.

53. Mitchell DW. Buddhism: Introducing the Buddhist experience. New York: Oxford University Press; 2002.

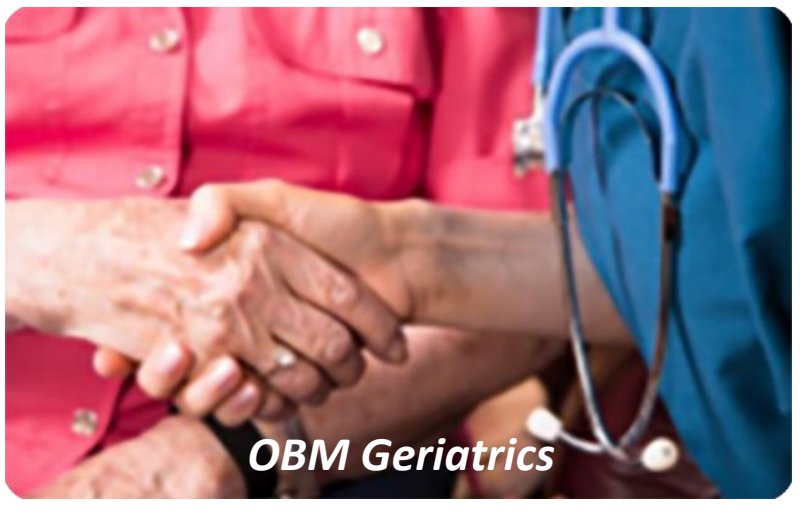

Enjoy OBM Geriatrics by:

1. Submitting a manuscript

2. Joining in volunteer reviewer bank

3. Joining Editorial Board

4. Guest editing a special issue

For more details, please visit: http://www.lidsen.com/journals/geriatrics 